\title{
Is subclinical hyperthyroidism a real syndrome, different from overt hyperthyroidism?
}

\author{
Raffaele Giubbini $\mathbb{i}^{1} \cdot$ Alessia Peli $^{1}$
}

Received: 2 November 2016 / Accepted: 6 November 2016

(C) Springer Science+Business Media New York 2016

Thyroid hormones affect the heart and cardiovascular function through many direct and indirect mechanisms. Symptoms related to the cardiovascular effects are very common and they are generally the first warning signs, alarming the patient and requiring diagnosis and treatment. Ertek and Cicero [1] have recently and extensively reviewed the relationship between hyperthyroidism and cardiovascular complications. In synthesis, in hyperthyroid patients, we can observe increased stroke volume due to increased preload (mainly related to sodium retention) and decreased afterload due to decreased systemic vascular resistance and, finally, increased myocardial contractility and heart rate related to sympathetic system activation. Sinusal tachycardia, atrial fibrillation and tachyarythmias, widened blood pressure and decreased exercise tolerance are common symptoms. Ischemic heart disease can be precipitated in patients with underlying diseases and thyrotoxic cardiomyopathy can occur in end stage, poorly treated patients.

Kaminski $\mathrm{G}$ et al. [2] have recently shown that patients with subclinical hyperthyroidism had lower exercise tolerance, larger end diastolic and end systolic volume indexes, and increased stroke volume, positively correlated to thyroid hormones concentrations. According to the results of their study, they conclude that cardiovascular alterations are common in subclinical hyperthyroidism. Subclinical hyperthyroidism is a definition, but not a syndrome with specific clinical features. This is not a semantic distinction but refers to the difficulty of justifying the presence of

Raffaele Giubbini

raffaele.giubbini@unibs.it

1 Chair of Nuclear Medicine, University of Brescia, Brescia, Italy suppressed thyroid-stimulating hormone (TSH) levels in presence of FT3 and FT4 values within the normal range. The confusion derives from the intrinsically equivocal method for defining a normal range. A reference range is defined as the prediction interval containing the $95 \%$ of values of a reference group [3]. In such a way, $2.5 \%$ of sample values will be less than the lower limit and $2.5 \%$ larger than the upper limit. Assuming a normal distribution, the $95 \%$ prediction interval can be defined as the interval limited by two standard deviations from either side of the population mean. In this case, the thresholds values identify three discrete intervals of thyroid hormones levels, which discriminate normals from patients with hypo or hyperthyroidism. This distinction is somewhat artificial as in the global population the distribution of blood concentrations of thyroid hormones is not a discrete but a continuous variable and the median value of patients with so called "subclinical hyperthyroidism" would be certainly different from that of a reference population. The results of the study of Kaminski $\mathrm{G}$ et al. reinforce the concept that patients with suppressed TSH but FT4 and FT3 in the normal range show similar cardiovascular parameters as in overt hyperthyroidism and for this reason they have decided to adequately treat their patients with normalization of indexes of cardiac function, avoiding further cardiovascular complications. Atrial fibrillation frequently occurs in older patients. In people over 60 years of age subclinical hyperthyroidism is associated with tripled risk of atrial fibrillation during a 10-year follow-up period [4] and increased cardiovascular mortality [5] as underlined in the discussion of the paper of Kaminski $\mathrm{G}$ et al.

A major concern that could be raised on their study is the methodology they have adopted for evaluating left ventricular function. Their patients underwent stress/rest 
myocardial perfusion imaging with $740 \mathrm{MBq}$ of $99 \mathrm{~m}$ technetium labeled compounds delivering an effective dose of $12 \mathrm{mSv}$, without adopting technologies for dose containment. According to the Choosing Wisely campaign, endorsed by the American Society of Nuclear Cardiology, physicians should not perform cardiac imaging for patients who are at low risk of ischemic heart disease and, in any case, they should use, whenever possible, methods to reduce radiation exposure, including not performing such tests when limited benefits are likely [6]. No patient of the study group had an imaging test positive for myocardial ischemia and all the functional parameters reported in the study could have been obtained by alternative methods, like ultrasound, without exposing the patients to ionizing radiations. It is therefore advisable that the procedure adopted in this study, justified for research purposes, will be not part of routine evaluation of subjects with "subclinical hypothyroidism".

\section{Compliance with ethical standards}

Conflict of interest The authors declare that they have no competing interests.

\section{References}

1. S. Ertek, A.F. Cicero, Hyperthyroidism and cardiovascular complications: a narrative review on the basis of pathophysiology. Arch. Med. Sci. 9(5), 944-952 (2013)

2. G. Kaminski, M. Dziuk, E. Szczepanek-Parulska, A. Zybek-Kocik, M. Ruchala, Electrocardiographic and scintigraphic evaluation of patients with subclinical hyperthyroidism during workout. Endocrine 53, 512-519 (2016)

3. S.K. Bangert, W.J. Marshall, W. Leonard, Clinical biochemistry: metabolic and clinical aspectsChurchill Livingstone/Elsevier, Philadelphia (2008)

4. C.T. Sawin, A. Geller, P.A. Wolf, A.J. Belanger, E. Baker, P. Bacharach, P.W. Wilson, E.J. Benjamin, R.B. D'Agostino, Low serum thyrotropin concentrations as a risk factor for atrial fibrillation in older persons. N. Engl. J. Med. 331(19), 1249-1252 (1994)

5. J.V. Parle, P. Maisonneuve, M.C. Sheppard, P. Boyle, J.A. Franklyn, Prediction of all-cause and cardiovascular mortality in elderly people from one low serum thyrotropin result: a 10-year cohort study. Lancet 358(9285), 861-865 (2001)

6. American Society of Nuclear Cardiology, Five things physicans and patients should question, Released 4 April 2012 